\title{
Erratum to: Effect of Training Leading to Repetition Failure on Muscular Strength: A Systematic Review and Meta-Analysis
}

\author{
Tim Davies $^{1} \cdot$ Rhonda Orr $^{1} \cdot$ Mark Halaki $^{1} \cdot$ Daniel Hackett $^{1}$
}

Published online: 18 February 2016

(c) Springer International Publishing Switzerland 2016

\section{Erratum to: Sports Med \\ DOI 10.1007/s40279-015-0451-3}

Page 1, abstract, Results, lines 29-40: The following three sentences, which previously read:

"Non-failure training resulted in a $0.6-1.3 \%$ greater strength increase than failure training. A small pooled effect favouring non-failure training was found ( $\mathrm{ES}=0.34 ; p=0.02$ ). Significant small pooled effects on muscular strength were also found for non-failure versus failure training with compound exercises $(\mathrm{ES}=0.37-0.38$; $p=0.03)$ and trained participants $(\mathrm{ES}=0.37$; $p=0.049$ ). A slightly larger pooled effect favouring nonfailure training was observed when volume-uncontrolled studies were included $(\mathrm{ES}=0.41 ; p=0.047)$. No significant effect was found for the volume-controlled studies, although there was a trend favouring non-failure training".

Should read:

"No significant strength increases were found for failure training versus non-failure training ( $\mathrm{ES}=0.05, p=0.73$ ). No significant effect was found when compound exercises (squat: $\mathrm{ES}=-0.03, p=0.91$; bench press: $\mathrm{ES}=-0.12$, $p=0.60)$ or isolated exercises $(\mathrm{ES}=0.23, p=0.34)$

The online version of the original article can be found under doi:10. 1007/s40279-015-0451-3.

Daniel Hackett

daniel.hackett@sydney.edu.au

1 Discipline of Exercise and Sport Science, Faculty of Health Sciences, The University of Sydney, 75 East Street, Lidcombe, Sydney, NSW 2141, Australia were included. No significant effect was found when volume was controlled or uncontrolled".

Page 1, abstract, Conclusion, lines 46-52: The following two sentences, which previously read:

"Overall, the results suggest that despite statistically significant effects on muscular strength being found for nonfailure compared with failure training, the small percentage of improvement shown for non-failure training is unlikely to be meaningful. Therefore, it appears that similar increases in muscular strength can be achieved with failure and non-failure training".

Should read:

"Overall, results suggest that similar increases in muscular strength can be achieved with failure and non-failure training”.

Page 6, columns 1-2, Sect. 3.3.1, lines 26-32: The following two sentences, which previously read:

"The differences in the change in muscular strength between non-failure and failure had small pooled ES values of 0.34 (95\% confidence interval $[\mathrm{CI}] 0.06-0.62$ ) and 0.33 (95\% CI 0.06-0.61) for analyses A and B, respectively. A statistically significant effect was found $(p=0.02)$ and favoured the non-failure group (Fig. 2)".

Should read:

"The differences in the change in muscular strength between non-failure and failure had a negligible pooled ES of -0.04 (95\% CI: -0.24 to 0.32 ) and 0.05 (95\% CI: -0.23 to 0.33 ) for analyses A and B respectively. No statistically significant effect was found for these analyses ( $p=0.77$ and $p=0.73$ for analyses A and B respectively) (Fig. 2)". 
Fig. 2 Forest plot of the results of the meta-analysis. The black squares and error bars signify the standardised difference (Std diff) values in the means (effect size) and $95 \%$ confidence interval $(C I)$ values, respectively. The black diamonds represent the pooled effect sizes. $d f$ degrees of freedom
Study name

Volume controlled

Drinkwater et al. [14]

Folland et al. [35]

Izquierdo et al. [31]

Rooney et al. [32]

Mean effect

Volume uncontrolled

Izquierdo-Gabarren et al. [37]

Sampson and Groeller [36]

Sanborn et al. [44]

Mean effect

Mean effect, total
Kramer et al. [38]
Std diff in means and $95 \%$ CI

Relative weight (\%)

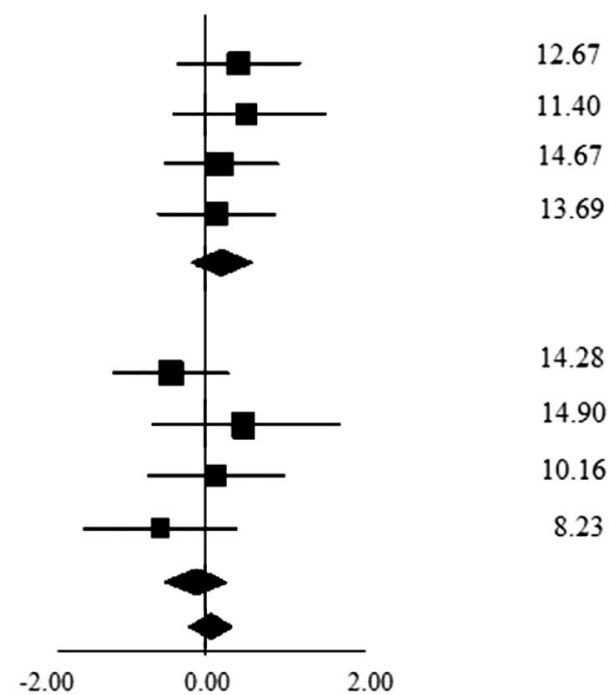

Favours non-failure Favours failure

Volume controlled

Heterogeneity: $\operatorname{tau}^{2}=0, \mathrm{chi}^{2}=1.69, \mathrm{df}=3(\mathrm{p}=0.64), \mathrm{I}^{2}=0 \%$; test for overall effect $Z=0.89(\mathrm{p}=0.37)$

Volume uncontrolled

Heterogeneity: tau ${ }^{2}=0.08, \mathrm{chi}^{2}=4.33, \mathrm{df}=3(\mathrm{p}=0.23), \mathrm{I}^{2}=30.64 \%$; test for overall effect $Z=-0.48(\mathrm{p}=0.64)$

Combined

Heterogeneity: $\operatorname{tau}^{2}=0, \mathrm{chi}^{2}=6.9, \mathrm{df}=7(\mathrm{p}=0.43), \mathrm{I}^{2}=0 \%$; test for overall effect $Z=0.29(\mathrm{p}=0.77)$
Page 6, column 2, Sect. 3.3.1, paragraphs 1 and 2, lines 32-56; page 7, column 1, paragraph 2, lines 1-4: The following seven sentences, which previously read:

"The subgroup analysis found that the effect was similar when the analysis was restricted to studies that used compound exercises $(n=5) \quad[\mathrm{ES}=0.38,95 \%$ CI $0.03-0.73, p=0.03$ for analysis $\mathrm{A}$; $\mathrm{ES}=0.37,95 \% \mathrm{CI}$ $0.03-0.72, p=0.03$ for analysis B]. Analysis of the types of compound exercises used (squat versus bench press) led to a non-significant effect (squat: $\mathrm{ES}=0.34,95 \% \mathrm{CI}$ -0.11 to $0.80, p=0.14$; bench press: $\mathrm{ES}=0.36,95 \% \mathrm{CI}$ -0.07 to $0.80, p=0.10$ ). When only studies that used isolated exercises were included (i.e. bicep curl and leg extension), a non-significant effect was also found $(\mathrm{ES}=0.23,95 \% \mathrm{CI}-0.24$ to $0.70, p=0.34)$. No significant effect was found between failure and non-failure training $(23.2 \%$ versus $18.3 \%$ increases in muscular strength, respectively) when only upper body exercises were analysed ( $\mathrm{ES}=0.28,95 \% \mathrm{CI}-0.07$ to 0.62 , $p=0.12$ ). Additionally, no significant effect was found for failure versus non-failure training when only lower body exercises were included; however, there was a trend towards greater increases in muscular strength following non-failure training $(\mathrm{ES}=0.37,95 \%$ CI $0.03-0.76$, $p=0.07)$.

Training status was found to produce a significant effect, with greater muscular strength gains in trained participants following non-failure training in analysis A (trained: $\mathrm{ES}=0.37,95 \%$ CI $0.001-0.75, p=0.049$; untrained: $\mathrm{ES}=0.30,95 \% \mathrm{CI}-0.12$ to $0.73, p=0.16$ ). Statistical significance was just missed in analysis $\mathrm{B}(\mathrm{ES}=0.36$, $95 \%$ CI -0.01 to $0.73, p=0.06) "$.

Should read:

"The subgroup analysis found a similar non-significant negligible effect when restricted to studies that used compound exercises $(n=5)(\mathrm{ES}=-0.06,95 \% \mathrm{CI}:-0.41$ to $0.29, p=0.73$, ES $=-0.05,95 \% \mathrm{CI}:-0.40$ to 0.30 , $p=0.78$ for analyses A and B respectively). Analysing the types of compound exercises used (squat versus bench press) also led to a non-significant effect (squat: ES = $-0.03,95 \%$ CI: -0.48 to $0.43, p=0.91$; bench press: $\mathrm{ES}=-0.12,95 \% \mathrm{CI}:-0.55$ to $0.32, p=0.60)$. When including studies that only used isolated exercises (i.e. bicep curl and leg extension) a non-significant effect was found $(\mathrm{ES}=0.23,95 \% \mathrm{CI}:-0.24$ to $0.70, p=0.34)$. No 
significant effect was found between failure and non-failure training $(23.2 \%$ versus $18.3 \%$ increase in muscular strength, respectively) when only upper body exercises were analysed ( $\mathrm{ES}=-0.03,95 \% \mathrm{CI}$ : -0.37 to 0.32 , $p=0.88$ ). Additionally, no significant effect was found for failure versus non-failure training when only lower body exercises were included ( $\mathrm{ES}=-0.03,95 \% \mathrm{CI}$ : -0.48 to $0.43, p=0.91)$.

Training status did not influence the muscular strength gains following failure versus non-failure training for analysis A (trained: $\mathrm{ES}=0.02,95 \% \mathrm{CI}:-0.35$ to 0.39 , $p=0.92$ and untrained: $\mathrm{ES}=-0.04,95 \% \mathrm{CI}:-0.34$ to $0.41, p=0.86)$ and for analysis $\mathrm{B}(\mathrm{ES}=0.07,95 \% \mathrm{CI}$ : -0.36 to $0.49, p=0.75)$ ".

Page 7, column 2, Sect. 3.3.1, paragraph 2, lines 6-9: The following sentence, which previously read:

"Both the funnel plot and Kendall's $\tau$ statistic $(\tau=0.18$, $p=0.54$ for analysis A; $s=0.32, p=0.27$ for analysis B) did not reveal publication bias in any study".

Should read:

"Both the funnel plot and Kendall's $\tau$ statistic $(\tau=0.11$, $p=0.71$ for analyses A and B) did not reveal publication bias for any study".

Page 9, column 1, Sect. 3.3.2, lines 6-13: The following sentence, which previously read:

"The differences in the change in muscular strength between non-failure and failure had small pooled ES values of 0.27 (95\% CI -0.11 to 0.65$)$ and 0.26 (95\% CI -0.12 to 0.64$)$ in analyses A and B, respectively. No statistically significant effect was found in analyses A and B $(p=0.15$ and $p=0.18$, respectively), although there was a trend favouring non-failure training (Fig. 2)".

Should read:

"The differences in the change in muscular strength between failure and non-failure had a negligible pooled ES of 0.18 (95\% CI: -0.21 to 0.56 ) and 0.19 (95\% CI: -0.19 to 0.58 ) for analyses A and B respectively. No statistically significant effect was found ( $p=0.37$ and $p=0.33$ ) for analyses A and B respectively (Fig. 2)”.

Page 9, column 1, Sect. 3.3.2, lines 15-18: The following sentence, which previously read:

"Both the funnel plot and Kendall's $\tau$ statistic ( $\tau=0.50$, $p=0.31$ for analysis $\mathrm{A} ; \tau=0.83, p=0.09$ for analysis B) did not reveal publication bias in any study".
Should read:

"Both the funnel plot and Kendall's $\tau$ statistic ( $\tau=0.09$, $p=0.83$ for analyses A and B) did not reveal publication bias for any study".

Page 9, column 1, Sect. 3.3.3, line 24; page 9, column 2, Sect. 3.3.3, lines 25-35: The following five sentences, which previously read:

"The differences in the change in muscular strength between non-failure and failure had a small ES of 0.41 (95\% CI 0.01-0.82). A statistically significant effect was found $(p=0.047)$ and favoured the non-failure group (Fig. 2). When studies that used only compound exercises were analysed $(n=3)$, a slightly greater significant effect was found, favouring the non-failure group ( $\mathrm{ES}=0.49$, $95 \%$ CI $0.03-0.95, p=0.04)$. The heterogeneity of the effect of failure versus non-failure training on muscular strength was zero $\left(I^{2}=0 \%\right)$. Both the funnel plot and Kendall's $\tau$ statistic $(\tau=0.17, p=0.73)$ did not reveal publication bias in any study".

Should read:

"The differences in the change in muscular strength between failure and non-failure had a negligible ES of -0.12 (95\% CI: -0.61 to 0.37 ). No statistically significant effect was found ( $p=0.64$ ) (Fig. 2). When studies that only used compound exercises were analysed $(n=3)$, no significant effect was found (ES $=-0.17,95 \% \mathrm{CI}:-0.63$ to $0.29, p=0.47)$. The heterogeneity of the effect of failure versus non-failure training on muscular strength was low $\left(I^{2}=30.64 \%\right)$ ".

Page 9, column 2, Sect. 4, lines 43-48; page 10, column 1, Sect. 4, lines 1-15: The following seven sentences, which previously read:

"The data show that despite both practices increasing muscular strength, non-failure training was found to be slightly more effective (i.e. there was a small effect). However, the effectiveness of non-failure training was influenced by training volume, training status and exercise type. A significant small effect of non-failure training on muscular strength remained only when studies that did not control for training volume were analysed. For studies that controlled for training volume, there was no significant effect on muscular strength between failure and non-failure training. Significant increases in muscular strength were found following non-failure training in individuals with previous training experience compared with novices, and for interventions that used compound exercises compared with 


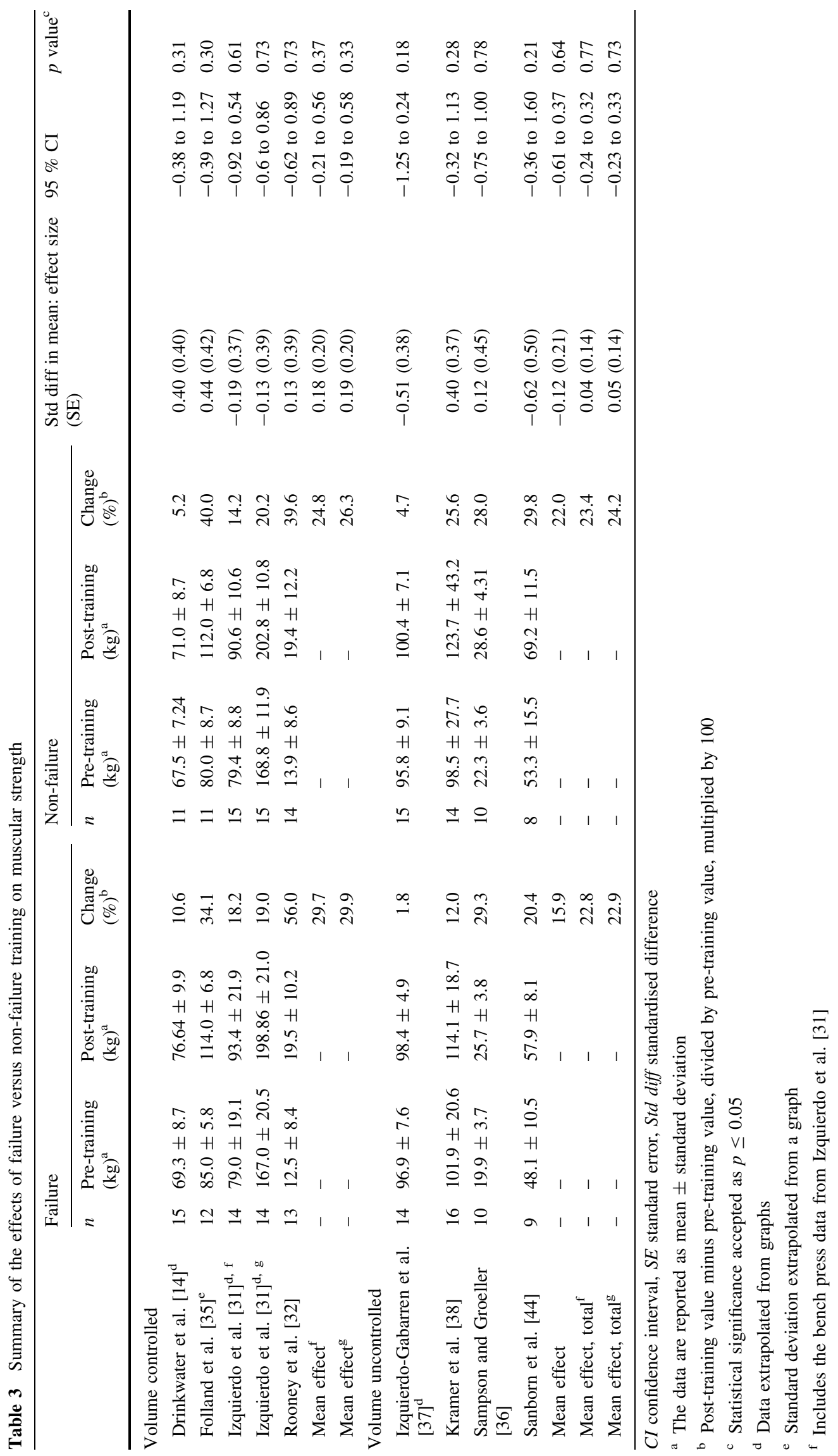


isolated exercises. Additionally, there was a slightly stronger effect favouring non-failure training compared with failure training on muscular strength in volume-uncontrolled studies that used only compound exercises. The heterogeneity of the effects for all meta-analyses was equal to zero, suggesting that all of the studies examined the same effect".

Should read:

"The data show that similar increases in muscular strength can be achieved with both practices. This result was not influenced by training volume, training status, and exercise type. The heterogeneity of effects for all meta-analyses was low suggesting that studies examined the same effect".

Page 12, column 1, Sect. 4.3, paragraph 1, lines 8-20: The following four sentences, which previously read:

"The findings from this review suggest that the effectiveness of failure versus non-failure training for increasing muscular strength may depend on which exercise is performed. Compound resistance exercises, which are considered to be superior to isolated exercises for increasing muscular strength [77], showed greater strength gains following nonfailure compared with failure training. An explanation for this result may relate to the increased demands of performing compound exercises compared with isolated exercises. Compound exercises place greater stress on the neuromuscular system because of the greater muscle groups that are stimulated and thus greater loads that are lifted [1]".

Should read:

"Compound resistance exercises, which are considered to be superior for increasing muscular strength compared to isolated exercises [77], showed similar gains following failure and non-failure training. This result was surprising as compound exercises place greater stress on the neuromuscular system due to the greater muscle groups stimulated and thus greater loads lifted [1]".

Page 12, column 2, Sect. 4.3, paragraph 2, lines 35-43: The following two sentences, which previously read:

"The results from the subgroup analysis also showed a trend towards greater gains in muscular strength with lower body exercise than with upper body exercise following non-failure training $(p=0.07)$. Like the results of compound exercises, this may have resulted from the increased demands/skill requirements of lower compared with upper body exercises and led to lesser muscular strength gains in the failure group, because of fatigue-related factors".

Should read:

"However, the subgroup analysis showed no difference between groups in strength gains for lower body compared to upper body training. This suggests that the increased demands/skill requirement of an exercise do not impact upon muscular strength development following failure or non-failure training".

Page 12, column 1, Sect. 4.3, paragraph 3, lines 50-53; page 12, column 2, Sect. 4.3, paragraph 3, lines 54-64: The following four sentences, which previously read:

"Therefore, a more probable explanation for the superior strength gains following non-failure compared with failure training with compound exercises (and the trend for lower body exercises) may be related to post-session recovery. Participants performing non-failure training could have recovered faster than participants performing failure training. This may have led towards a greater rate of progress (i.e. loading and training stimulus) and adaptation in the non-failure group, and thus greater strength gains. Furthermore, because of the average exercise intervention lasting approximately 9 weeks, the ability to recover and progressively overload following subsequent training sessions over this relatively short duration would likely have a positive influence on strength gains".

Should read:

"It could be argued that less fatigue following non-failure training compared to failure training may lead to a greater rate of progress (i.e. may allow for greater increments in loading). Therefore, the effectiveness of failure versus nonfailure training on muscular strength, at least in the shortterm (i.e. 6 weeks) may be influenced by the ability to recover and allow for progressive overload. This may have implications for resistance trainers based on their level of performance and experience".

Page 12, column 2, Sect. 4.4, lines 76-80: The following sentence, which previously read:

"However, the findings from this review showed that trained participants responded more favourably to nonfailure compared with failure training $(\sim 14$ and $\sim 12 \%$, respectively), suggesting that regular failure training may be too demanding for strength athletes".

Should be deleted.

Page 12, column 2, Sect. 4.4, lines 84-92: The following two sentences, which previously read:

"In this review, even though failure training did increase the muscular strength of the trained participants, it appears that too great a training demand may not optimise muscular strength development. For untrained participants, the similar increases in muscular strength for failure versus nonfailure training ( $\sim 34 \%$ increase for both groups) suggests 
that subtle differences in resistance training prescription may not have a large impact on muscular strength".

Should read:

"In this review, untrained participants showed similar increases in muscular strength for failure versus non-failure training ( $\sim 34 \%$ increase for both groups) suggests that subtle differences in resistance training prescription may not have a large impact on muscular strength".

Page 13, column 2, Sect. 4.6, lines 57-60: The following sentence, which previously read:

"While this review offers quantitative evidence to support the efficacy of non-failure compared with failure training for muscular strength development, there are certain limitations that should be discussed".

Should read:

"While this review offers quantitative evidence showing no difference between failure and non-failure training in the development of muscular strength, there are certain limitations that should be discussed".

Page 14, column 1, Sect. 5, lines 20-22: The following sentence, which previously read:

"Training volume, resistance training experience and type of exercise were shown to have an impact on muscular strength following failure and non-failure training".

Should read:

"Training volume, resistance training experience, and type of exercises were shown not to have an impact on muscular strength following failure and non-failure training".

Page 8, Table 3: This table (Table 3) should read:

Page 9, Fig. 2: This figure (Fig. 2) should read: 